\title{
Response of turbulent enstrophy to sudden implementation of spanwise wall oscillation in channel flow*
}

\author{
Mingwei GE ${ }^{1, \dagger}$, Guodong $\mathrm{JIN}^{2}$ \\ 1. School of Renewable Energy, North China Electric Power University, Beijing 102206, China; \\ 2. State Key Laboratory of Nonlinear Mechanics, Institute of Mechanics, Chinese \\ Academy of Sciences, Beijing 100080, China
}

\begin{abstract}
The response of turbulent enstrophy to a sudden implementation of spanwise wall oscillation (SWO) is studied in a turbulent channel flow via direct numerical simulation. In the beginning of the application of SWO, a significant correlation is formed between $\omega_{y}^{\prime}$ and $\omega_{z}^{\prime}$. A transient growth of turbulent enstrophy occurs, which directly enhances turbulent dissipation and drifts the turbulent flow towards a new lower-drag condition. Afterwards, the terms related to the stretching of vorticity $\left(\omega_{x}, \omega_{y}^{\prime}\right.$, and $\left.\omega_{z}\right)$, the inclination of $\omega_{y}^{\prime}$ by $\frac{\partial w}{\partial y}$, the turn of $\bar{\omega}_{z}$ by $\frac{\partial v^{\prime}}{\partial z}$, and the horizontal shear of $\bar{\omega}_{z}$ by $\frac{\partial w^{\prime}}{\partial x}$ are suppressed due to the presence of SWO, leading to attenuation of the turbulent enstrophy.
\end{abstract}

Key words turbulent channel flow, transport of enstrophy, drag reduction, spanwise wall oscillation (SWO)

Chinese Library Classification O357.5

2010 Mathematics Subject Classification $\quad 76 \mathrm{~F} 40$

\section{Introduction}

Drag reduction is an important issue in fluid mechanics, which has attracted more and more interest recently owing to its potential of decreasing energy consumption in industry. Generally, drag reduction control can be divided into active control and passive control. Passive control, which keeps the control measures constant during the work, does not require energy input from the external environment and is very convenient for application such as the riblets ${ }^{[1-3]}$ and hydrophobic surface ${ }^{[4-7]}$. However, most of them are criticized for their low drag reduction rate and bad environmental adaptability. Active control is developed to avoid the disadvantages of passive control. It can obtain very substantial drag reduction but needs additional energy input. Furthermore, active control can be grouped into feedback control which needs sophisticated feedback control systems, and open-loop control which needs additional energy input without feedback. In this paper, open-loop active control is mainly concerned.

* Received Oct. 3, 2016 / Revised Dec. 21, 2016

Project supported by the National Natural Science Foundation of China (Nos.11402088 and 51376062), the Opening Fund of State Key Laboratory of Nonlinear Mechanics, and the Fundamental Research Funds for the Central Universities (No. 2107MS022)

$\dagger$ Corresponding author, E-mail: gmwncepu@163.com 
For the open-loop active control schemes, the spanwise wall oscillation has been extensively studied due to its significant drag reduction rate ${ }^{[8-11]}$. The suppression of turbulence by spanwise wall oscillation (SWO) in a canonical channel flow was first investigated by Jung et al. ${ }^{[12]}$ via direct numerical simulation, and a $40 \%$ frictional drag reduction was obtained. Soon afterwards, the numerical results were verified by Laadhari et al. ${ }^{[13]}$ and Choi et al.[14] in a turbulent boundary layer through physical experiments. Through direct numerical simulation of a pipe flow with circumferential wall oscillations, Quadrio and Sibilla ${ }^{[15]}$ attributed the drag reduction to the tangential advection arising from the Stokes layer. After a similar simulation, Choi and Graham $^{[16]}$ claimed that the oscillations affect the relations between the near-wall streamwise vortices and low- and high-speed fluids, and then suppress the production of Reynolds shear stress. Focusing on the initial behavior of the Reynolds stress to the harmonic oscillations, $\mathrm{Xu}$ and Huang ${ }^{[17]}$ found that the motions of the wall attenuate the distribution term in the transport equations and finally suppress the turbulence. Different from the analyses of the long-term drag reduced condition, the study of the response of the wall bounded turbulent flow to a sudden spanwise wall oscillation can provide more details of the flow evolution ${ }^{[18]}$. Recently, Ricco et al. ${ }^{[19]}$ studied the global turbulent enstrophy to the wall oscillation in a very short time in a turbulent channel flow. They found that in the beginning stage, after a sudden implementation of spanwise oscillations, the turbulent enstrophy shows a transient increase which directly enhances the turbulent dissipation. As a consequence, the activity of turbulence is suppressed by the transient increase of the turbulent enstrophy in the initial phase, which drifts the turbulent flow towards a new lower-drag condition after a long time. The understanding of the mechanism of drag reduction by the harmonic wall oscillations has been greatly pushed forward by the efforts of many scientists. However, the whole picture of the mechanism for drag reduction is still not clear, and there are some problems not enclosed. In the work of Ricco et al. ${ }^{[19]}$, the transient growth of the turbulent enstrophy was emphasized. However, the reason for the transient growth as well as the evolution of the turbulent enstrophy after the transient growth is still not clear.

In this paper, the transport of the turbulent enstrophy is studied in detail in a turbulent channel flow subjected to sudden wall oscillations. The objective of this work is to gain a further insight into the mechanism for drag reduction by wall oscillations on the aspect of vortical dynamics. The transient growth and the transport of the turbulent enstrophy on the initial stage are analyzed.

\section{Numerical calculations and flow configuration}

\subsection{Numerical methods}

The turbulent flow between two infinite parallel flat plates with oscillating walls is studied through direct numerical simulation. The Navier-Stokes equations for the incompressible Newtonian fluid are taken as the governing equations,

$$
\begin{aligned}
& \frac{\partial u_{i}}{\partial t}+u_{i} \frac{\partial u_{i}}{\partial x_{i}}=-\frac{\partial p}{\partial x_{i}}+\frac{1}{R e_{\tau}} \frac{\partial^{2} u_{i}}{\partial x_{j} \partial x_{j}}, \\
& \frac{\partial u_{i}}{\partial x_{i}}=0
\end{aligned}
$$

where $u_{i}$ is the $i$ th component of the flow velocity, $t$ is the time, $\rho$ is the density of fluid, and $p$ is the pressure. All the flow variables are dimensionless through the wall frictional velocity $u_{\tau}$ and the kinematic viscosity of the fluid $\nu$, of which $u_{\tau}=\sqrt{\tau_{\mathrm{w}} / \rho}$. The computational domain and the coordinate system are shown in Fig. 1 . The Reynolds number $R e_{\tau}$ is based on $u_{\tau}$ and the half channel width $H$ is 180 . The periodic boundary condition is used in the streamwise and spanwise directions. The oscillation of the wall is introduced by the first type boundary 
condition,

$$
w_{\mathrm{w}}=W \sin (2 \pi t / T) .
$$

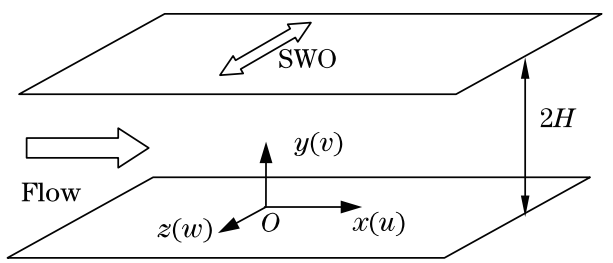

Fig. 1 Computational domain and coordinate system

Here, we select the amplitude of the oscillation $W=12$ and the cycle $T=100$ following the optimal case by Ricco et al. ${ }^{[19]}$. A pseudo-spectral method is used to solve the three-dimensional Navier-Stokes equations. The Fourier Galerkin and Chebyshev-Tau methods are used for spatial discretization of the channel flow, and a third-order time splitting method is adopted for advance of time. The direct numerical simulation method has been well validated by $\mathrm{Xu}$ el al. ${ }^{[20]}$ and Ge et al. ${ }^{[21]}$. The computational domain spans $4 \pi \times 2 \times 2 \pi$ in the streamwise, wall normal, and spanwise directions, respectively, in accordance with the $128 \times 129 \times 128$ grids. During numerical simulation, the pressure gradient is kept constant, and hence the flow rate increases with the evolution of turbulence due to the drag reduction.

\subsection{Flow field decomposition}

Before we proceed further, some types of average operators are introduced here. We assume that there is a flow quantity $f(x, y, z, t)$, and it can be decomposed as ${ }^{[22]}$

$$
f(x, y, z, t)=\langle f\rangle(y)+\tilde{f}(y, t)+f^{\prime}(x, y, z, t),
$$

where the variable with the tilde head denotes the periodic fluctuation due to the SWO, and the one with the superscript denotes the stochastic fluctuation. In this paper, the turbulent enstrophy is referred to as $\omega_{i}^{\prime} \omega_{i}^{\prime}$. The mean value of a quantity is defined as

$$
\langle f\rangle(y)=\frac{1}{n T} \int_{0}^{t=n T} \bar{f}(y, t) \mathrm{d} t,
$$

where

$$
\bar{f}(y, t)=\frac{1}{L_{x} L_{z}} \int_{0}^{L_{z}} \int_{0}^{L_{x}} f(x, y, z, t) \mathrm{d} x \mathrm{~d} z
$$

Hence,

$$
\begin{aligned}
& \tilde{f}(y, t)=\bar{f}(y, t)-\langle f\rangle(y), \\
& f^{\prime}(x, y, z, t)=f(x, y, z, t)-\bar{f}(y, t) .
\end{aligned}
$$

A global quantity for an instantaneous flow field is defined as

$$
[f](t)=\frac{1}{L_{x} L_{z}} \int_{0}^{L_{z}} \int_{0}^{H} \int_{0}^{L_{x}} f(x, y, z, t) \mathrm{d} x \mathrm{~d} y \mathrm{~d} z=\int_{0}^{H} \bar{f}(y, t) \mathrm{d} y .
$$

The comprehensive global quantity can be defined as

$$
[f]_{\mathrm{g}}(t)=\frac{1}{n T} \int_{0}^{n T}[f](t) \mathrm{d} t .
$$




\subsection{Basic flow statistics}

Figures 2(a) and 2(b) show time traces of the space-averaged streamwise skin friction and the flow rate after the application of SWO at $t=0$, respectively. In the early stage of the implementation of SWO, the skin friction reduces sharply, and hence the balance between the friction drag and the driven pressure is broken down, which then results in a quick growth of the flow rate of the turbulent channel. After a long time evolution, the turbulent channel flow achieves a new statistical steady state with lower drag coefficient. Noteworthily, under the constant pressure gradient, drag reduction does not show a real decrease of skin friction in the new statistical state. It manifests itself in terms of the increase of the flow rate. Following Kasagi et al. ${ }^{[23]}$, the skin friction coefficient is defined as $C_{\mathrm{f}}=2 / U_{\mathrm{b}}^{2}$, where $U_{\mathrm{b}}$ represents the bulk mean velocity, and the drag reduction rate is defined as the change of $C_{\mathrm{f}}$,

$$
r_{\mathrm{d}}=\frac{r_{\mathrm{f}}^{2}-r_{\mathrm{f} 0}^{2}}{r_{\mathrm{f}}^{2}} \times 100 \%
$$

where $r_{\mathrm{fo}}$ denotes the time and space averaged flow rate of the initial state, while $r_{\mathrm{f}}$ is the flow rate of the new state after the SWO. For the case in the present paper, a drag reduction about $31 \%$ is obtained, which shows good agreement with Ricco et al. ${ }^{19]}$, in which $R e_{\tau}$ was selected to be 200. Similar drag reductions were also obtained by Choi et al. ${ }^{[16]}$ and Ricco and $\mathrm{Wu}^{[24]}$ through physical experiments. Figure $2(\mathrm{c})$ shows the distribution of velocity fluctuations along $y^{+}$. As can be observed, the fluctuating velocities are only affected in the near wall region $y^{+}<40$. The maximum value of $\left\langle u^{\prime} u^{\prime}\right\rangle$ reduces about $30 \%$, and the peak value location is shifted from $y^{+} \approx 19$ to $y^{+} \approx 14$. Due to the SWO, $\left\langle w^{\prime} w^{\prime}\right\rangle$ is obviously enhanced near the wall. The maximum value of $\left\langle w^{\prime} w^{\prime}\right\rangle$ increases about $30 \%$, and its location is shifted closer to the wall. Different from the previous two components of velocity fluctuations, $\left\langle v^{\prime} v^{\prime}\right\rangle$ seems almost unchanged in the presence of SWO. Figure $2(\mathrm{~d})$ shows the vorticity fluctuations along $y^{+}$. Obviously, the vorticity fluctuations are also influenced by the SWO only in the near wall region $y^{+}<60$. In the near wall region, $\omega_{x}$ is substantially excited, while $\omega_{y}$ is obviously refrained. Compared with the previous two components, the change of $\omega_{z}^{\prime}$ is more complex. In the sublayer, $\omega_{z}^{\prime}$ is greatly suppressed, while in the region $5<y^{+}<60$, it is repressed to a lower degree.

When periodic oscillations are applied to the walls of the parallel flat channel, the physical problem can be simplified to the Stokes' second problem, and the spanwise velocity of the flow field can be analytically solved as ${ }^{[25]}$

$$
\bar{w}(y, t)=A \exp (-\sqrt{(\pi t / T)} y) .
$$

Figure 3 shows the phase-averaged spanwise velocity from both the present direct numerical simulation and the analytical solution obtained from Eq. (12). The numerical results agree well with the analytical results of the Stokes' problem. The results of the basic statistics shown here give a firm validation of the present numerical methods and also briefly show the response of the flow field to the SWO.

\section{Transient response of turbulent enstrophy}

Turbulent enstrophy is the criterion of the intensity of vorticity fluctuations, which plays an important role in the evolution of the turbulent flow. Moreover, in the case of turbulent channel flow with the SWO, the global turbulent enstrophy can be mathematically regarded as the equality of the global turbulent dissipation ${ }^{[19]}$. Hence, investigation of the transport of the turbulent enstrophy is of great importance to understand the mechanism of drag reduction due to the SWO. The turbulent enstrophy can be divided into three parts: $\omega_{x}^{\prime} \omega_{x}^{\prime}\left(e_{\mathrm{t} x}\right), \omega_{y}^{\prime} \omega_{y}^{\prime}\left(e_{\mathrm{t} y}\right)$, and $\omega_{z}^{\prime} \omega_{z}^{\prime}\left(e_{\mathrm{t} z}\right)$. Figure 4 shows the temporal evolution of the instantaneous global quantity of $e_{\mathrm{t} x}, e_{\mathrm{t} y}, e_{\mathrm{t} z}$ and the turbulent enstrophy. It is evident that in the beginning $t<50$, there is a 


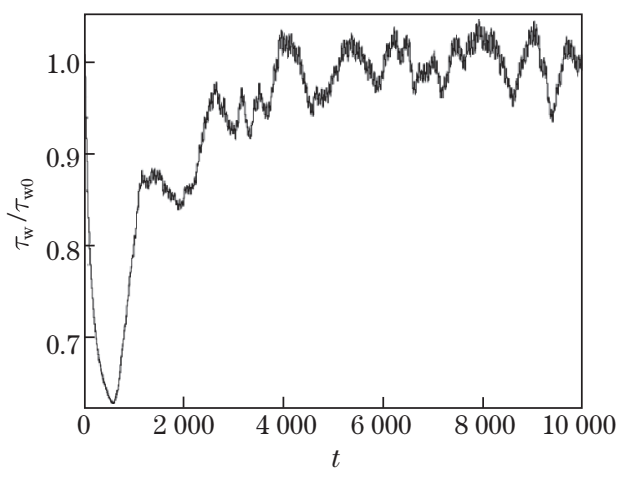

(a) Time traces of $\tau_{\mathrm{w}} / \tau_{\mathrm{w} 0}$ for turbulent channel flow after implementation of SWO

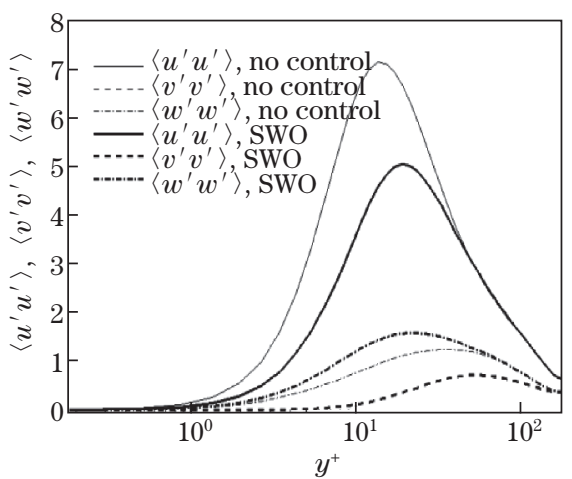

(c) Distribution of velocity fluctuations along $y^{+}$

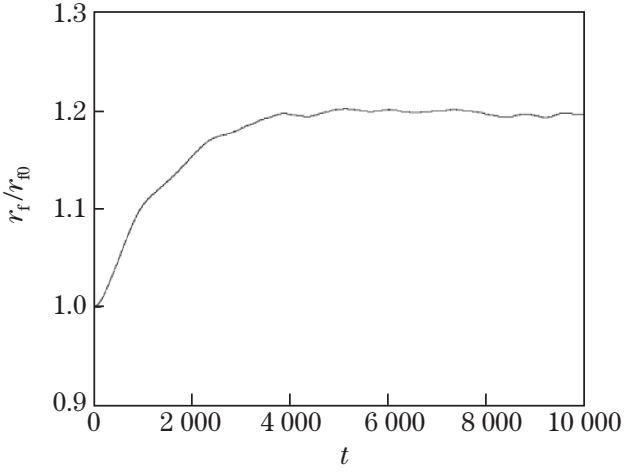

(b) Time traces of $r_{\mathrm{f}} / r_{\mathrm{f} 0}$ for turbulent channel flow after implementation of SWO

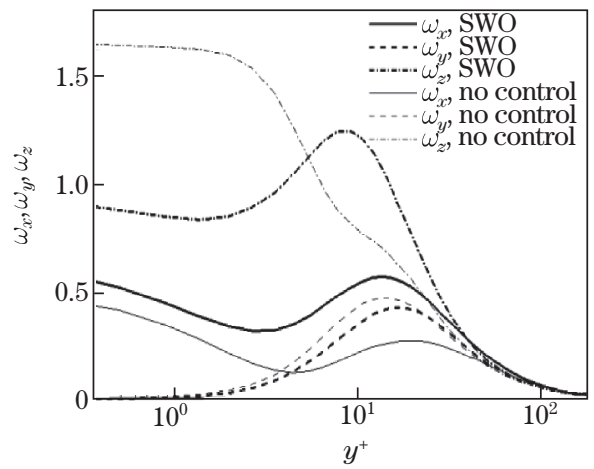

(d) Distribution of vorticity fluctuations along $y^{+}$

Fig. 2 Some basic statistics with SWO applied at $t=0$

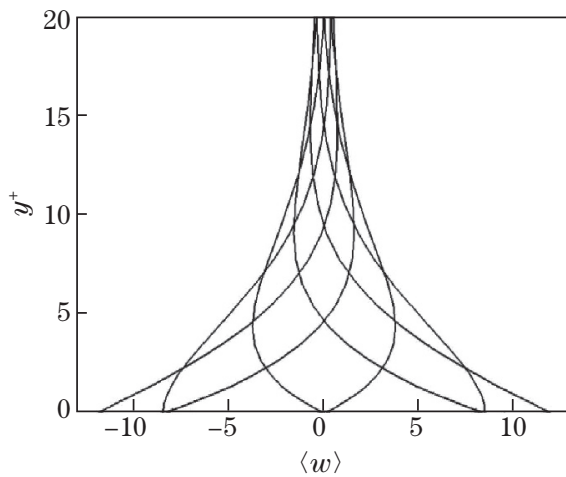

(a) Direct numerical simulation

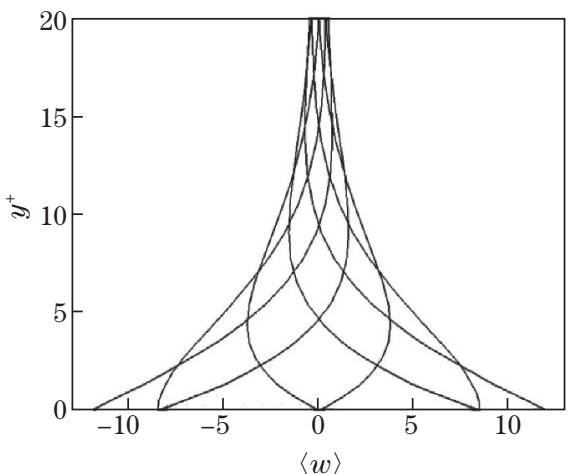

(b) Analytical solution

Fig. 3 Phase-averaged spanwise velocity for different phases

transient growth for turbulent enstrophy due to the SWO. This means that the dissipation which is mathematically equal to the global turbulent enstrophy increases transiently at the beginning after the application of SWO. As announced by Ricco et al. ${ }^{[19]}$, the activity of turbulence is dissipated by the initial increase of dissipated term and then set into a state with lower turbulent kinetic energy. The decrease of turbulent kinetic energy for the instantaneous channel flow means a laminarization which causes a rapid decrease of the Reynolds stress and results in a lower skin friction. Note that the flow rate keeps almost unchanged in such a short time. Hence, the flow is accelerated due to the constant pressure gradient, as shown in Fig. 2(b). As the mass 


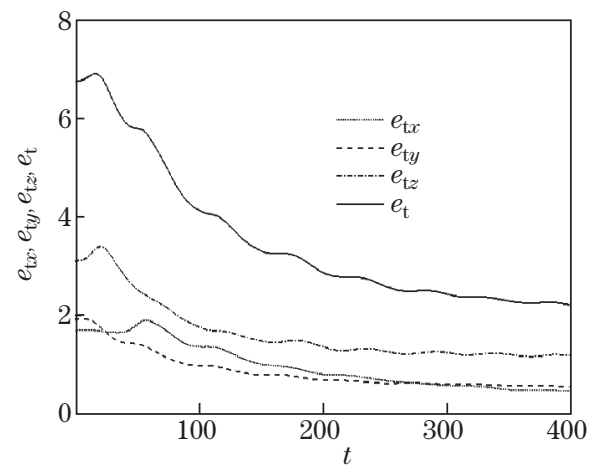

Fig. 4 Time traces of instantaneous global quantities of $e_{\mathrm{t} x}, e_{\mathrm{t} y}, e_{\mathrm{t} z}$, and $e_{\mathrm{t}}\left(e_{\mathrm{t}}\right.$ denotes turbulent enstrophy, and SWO starts at $t=0$ )

flow rate increases, the skin friction increases gradually. Finally, the pressure gradient and the skin friction achieve a new balance. At the new state, the friction drag normalized by its initial value varies around 1 (see Fig. 2(a)). Recall that, in the present case, the drag reduction does not show a real decrease of skin friction in the new quasi-steady state under the constant pressure gradient. Through a careful investigation on the energy balance of the channel flow with SWO, Ricco et al. ${ }^{[19]}$ found that a higher proportion of energy input by pressure gradient was dissipated by the viscous effect, and a lower proportion of the energy was dissipated by the turbulent effect compared with the canonical channel flow. The results indicate that the flow tends to be laminar with the decrease of $C_{\mathrm{f}}$ in the whole process. Obviously, the transient growth of turbulent enstrophy (dissipation) plays a very important role in the evolution of the turbulent flow after the application of the SWO. As shown in Fig. 4, the transient increase of turbulent enstrophy can be then attributed to the initial growth of $e_{\mathrm{t} z}$. Hence, in the next step, the response of the related terms in the transport equations of $e_{\mathrm{t} z}$ will be analyzed first. After that, the transport of the other two parts will be studied.

\subsection{Transport of $e_{\mathrm{t} z}$}

The transport equation for the global $e_{\mathrm{t} z}$ can be written as

$$
\begin{aligned}
\frac{\partial\left(\omega_{z}^{\prime} \omega_{z}^{\prime}\right)}{\partial t}= & 2 \underbrace{\left(\omega_{z}^{\prime} \omega_{y}^{\prime} \frac{\partial \bar{w}}{\partial y}\right)}_{P_{31}}+\underbrace{2\left(\omega_{z}^{\prime} \frac{\partial w^{\prime}}{\partial x} \frac{\partial \bar{w}}{\partial y}\right)}_{P_{32}}-\underbrace{2\left(\omega_{z}^{\prime} \frac{\partial w^{\prime}}{\partial z} \frac{\partial \bar{u}}{\partial y}\right)}_{P_{33}} \\
& +\underbrace{2\left(\omega_{z}^{\prime} \omega_{x}^{\prime} \frac{\partial w^{\prime}}{\partial x}\right)}_{P_{34}}+\underbrace{2\left(\omega_{z}^{\prime} \omega_{y}^{\prime} \frac{\partial w^{\prime}}{\partial y}\right)}_{P_{35}}+\underbrace{2\left(\omega_{z}^{\prime} \omega_{z}^{\prime} \frac{\partial w^{\prime}}{\partial z}\right)}_{P_{36}} \\
& +\underbrace{2\left(v^{\prime} \omega_{z}^{\prime} \frac{\partial^{2} \bar{u}}{\partial^{2} y}\right)}_{P_{37}}-\underbrace{2 \nu\left(\frac{\partial \omega_{z}^{\prime}}{\partial x_{j}} \frac{\partial \omega_{z}^{\prime}}{\partial x_{j}}\right)}_{D_{3}},
\end{aligned}
$$

where $P_{31}$ to $P_{37}$ denote the contribution to the global $e_{\mathrm{t} z}$ from the production terms of $\omega_{z}^{\prime} . P_{31}$, $P_{32}, P_{33}$, and $P_{37}$ are the production terms related to the average flow. $P_{31}$ is the contribution induced by the tilting of $\omega_{y}^{\prime}$ by the spanwise mean shear. $P_{32}$ is induced by the turn of $\bar{\omega}_{x}$ by $\frac{\partial w^{\prime}}{\partial x} . P_{33}$ is caused by the stretching of $\bar{\omega}_{z}$ by the fluctuating quantity $\frac{\partial w^{\prime}}{\partial z} \cdot P_{37}$ is an additional production term involving the second derivative of $\bar{u}$. The other three production terms $P_{34}, P_{35}$, and $P_{36}$ are caused by the stochastic fluctuations. $P_{34}$ and $P_{35}$ are attributed to the turn of $\omega_{x}^{\prime}$ and $\omega_{y}^{\prime}$, respectively, while $P_{36}$ is produced by the stretching of $\omega_{z}^{\prime}$. The last term in Eq. (13), $D_{3}$, is a global quantity from the viscous dissipation term. Compared 
with the transport equation of $e_{\mathrm{t} z}$ in the canonical turbulent channel flow, the newly appearing terms due to the spanwise oscillation, $P_{31}$ and $P_{32}$, are directly affected by the SWO since the phase-averaged spanwise velocity $\bar{w}$ explicitly appears in these terms.

\subsubsection{Transient increase of $P_{31}$ at beginning}

Time traces of the production terms of $e_{\mathrm{t} z}$ in Eq. (13) are shown in Fig. 5. The transport of $e_{\mathrm{t} z}$ is mainly dominated by $P_{31}, P_{32}, P_{33}$, and $P_{34}$. At the beginning, $P_{31}$ exhibits an acute increase after the SWO, while all the other terms change more insensitively. Attributed to the dominated role of $P_{31}$ in the transport of $e_{\mathrm{t} z}$, both the peak values of $e_{\mathrm{t}}$ and $e_{\mathrm{t} z}$ appear at about $t=20$, which is very close to that of $P_{31}$. Evidently, it is the prime increase of $P_{31}$ that results in the initial increase of $e_{\mathrm{t} z}$ and then $e_{\mathrm{t}}$. In other words, it is the tilting of $\omega_{y}^{\prime}$ by the spanwise mean shear that induces the transient increase of $e_{\mathrm{t}}$, and then represses the turbulent kinetic energy. To find the reason of the transient increase of $P_{31}$, the term can be decomposed into two parts, $\omega_{z}^{\prime}$ and $\omega_{y}^{\prime} \frac{\partial \bar{w}}{\partial y}$. The former one denotes the fluctuating spanwise vorticity in the

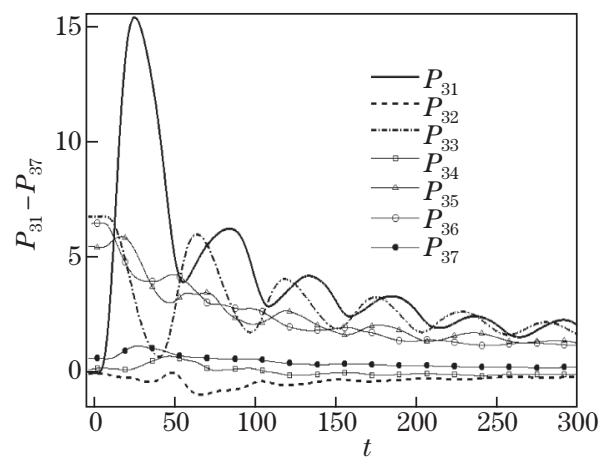

Fig. 5 Time traces of production terms of $e_{\mathrm{t} z}$ (SWO starts at $\left.t=0\right)$, where all terms are multiplied by scale factor of 100

flow field, while the latter one denotes the new $\omega_{z}^{\prime}$ generated by the tilting of $\omega_{y}^{\prime}$. Noteworthily, both parts are stochastic fluctuations with a space-average quantity zero. Thus, the high value of $P_{31}$ indicates that there is a high correlation between the two parts. The relationship between $\omega_{z}^{\prime}$ and the newly produced $\omega_{z}^{\prime}$ by the tilting of $\omega_{y}^{\prime}$ at $t=25$ on the plane of $y^{+}=10$ is shown in Fig. 6. An obvious positive correlation between $\omega_{z}^{\prime}$ and the newly produced $\omega_{z}^{\prime}$ in the flow filed can be found due to the mean spanwise shear. Furthermore, the correlation between the two parts can be attributed to the local correlation between $\omega_{z}^{\prime}$ and $\omega_{y}^{\prime}$ since $\frac{\partial \bar{w}}{\partial y}$ is constant on the whole plane.

Figure 7 shows the evolution of the correlation between $\omega_{z}^{\prime}$ and $\omega_{y}^{\prime}$. In the flat channel before the SWO, $\omega_{z}^{\prime}$ and $\omega_{y}^{\prime}$ are two separate quantities. However, due to the effect of SWO, a substantial negative correlation forms at $t=T / 8$. With the increase of the spanwise wall shear, the correlation increases to a higher degree at $t=T / 4$. The results show that under the negative spanwise shear stress, the positive $\omega_{z}^{\prime}$ tends to appear at the location where $\omega_{y}^{\prime}$ is negative, and the negative $\omega_{z}^{\prime}$ tends to appear at the location where $\omega_{y}^{\prime}$ is positive. Figure $7(\mathrm{~d})$ shows the schematic of the mechanism of the correlation. Assume a position in the flow filed with a positive $\omega_{y}^{\prime}$ due to the SWO. A new negative $\omega_{z}^{\prime}$ will be produced by the effect of $\omega_{y}^{\prime}$ and the negative $\frac{\partial \bar{w}}{\partial y}$. Hence, $\omega_{z}^{\prime}$ at the position with a positive $\omega_{y}^{\prime}$ will be reduced, which makes the point shift to the negative direction in the coordinate of $\omega_{z}^{\prime}-\omega_{y}^{\prime}$. Correspondingly, the points in the third and fourth quadrants move to the positive direction. At $t=3 T / 4$, the same 


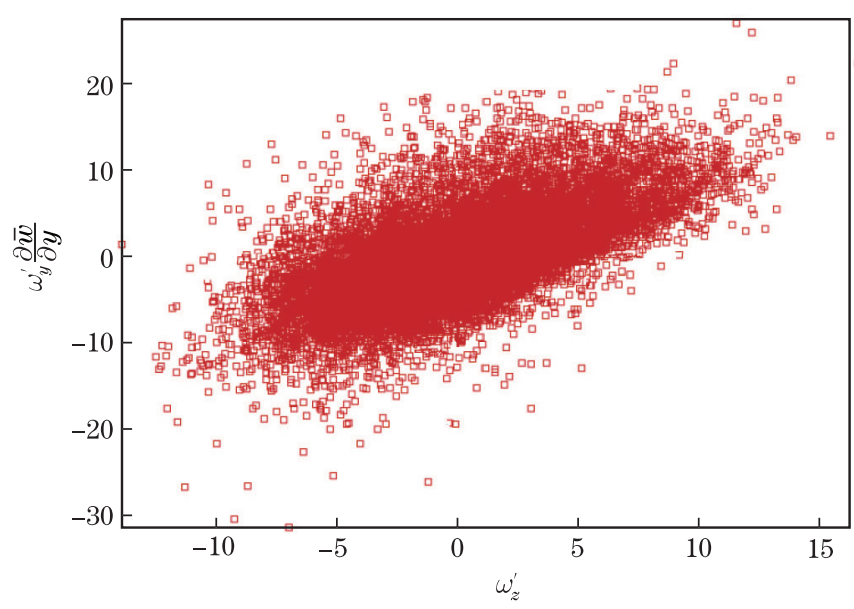

Fig. 6 Relationship between $\omega_{z}^{\prime}$ and $\omega_{y}^{\prime} \frac{\partial \bar{w}}{\partial y}$ at $t=25$

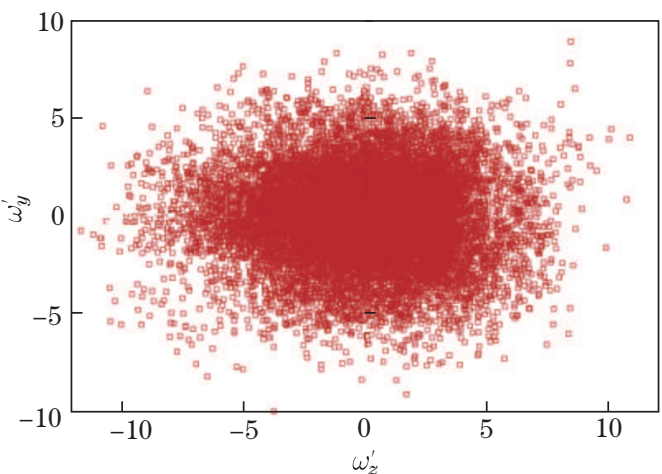

(a) $t=0$

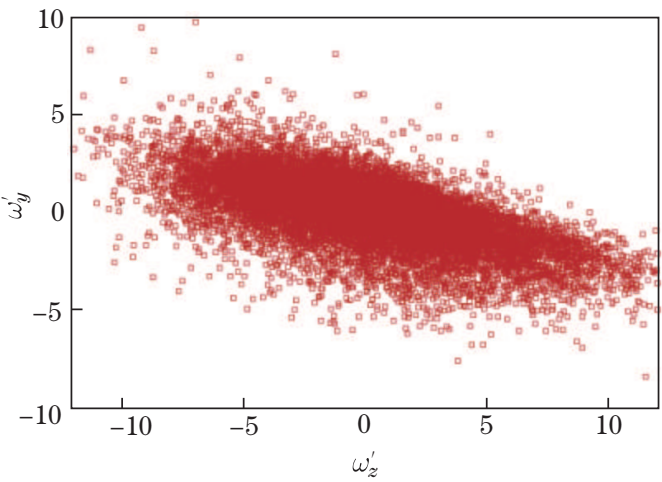

(c) $t=25$

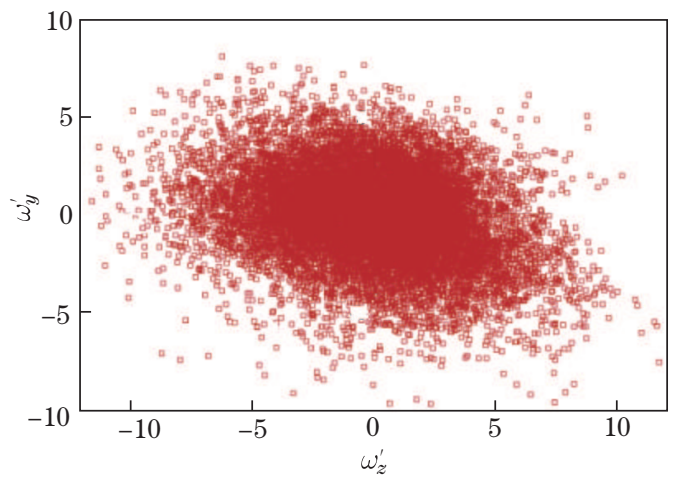

(b) $t=12.5$

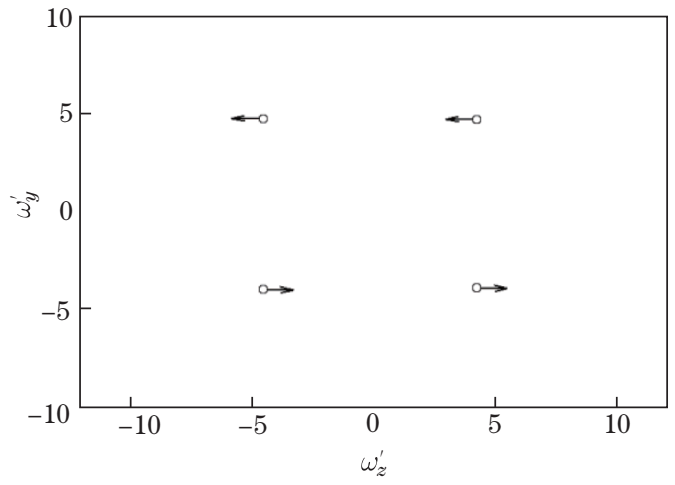

(d) Schematic of formation of correlation between $\omega_{z}^{\prime}$ and $\omega_{y}^{\prime}$

Fig. 7 Evolution of correlation between $\omega_{z}^{\prime}$ and $\omega_{y}^{\prime}$

mechanism also works, and a positive correlation between $\omega_{z}^{\prime}$ and $\omega_{y}^{\prime}$ is induced. By now, the production of the correlation between $\omega_{z}^{\prime}$ and $\omega_{y}^{\prime}$ is unfolded to give an explanation to the transient increase of $P_{31}$. Although the formation of the correlation is very simple, it shows a clear role of the spanwise shear in the drag reduction. 


\subsubsection{Attenuation of $e_{\mathbf{t} \boldsymbol{z}}$ after beginning}

After the transient increase, $e_{\mathrm{t} z}$ decreases dramatically, and the attenuation is beyond $50 \%$ at $t=400$. As is shown in Fig. 5 , the attenuation of $e_{\mathrm{t} z}$ can be mainly attributed to the inhibition of the terms $P_{31}, P_{33}, P_{35}$, and $P_{36}$. It should be pointed out that although the term $P_{31}$ is a new term compared with the initial state, the rapid minimizing of this term still makes important sense. Assume that, if the term $P_{31}$ keeps the peak value at $t=20$ in the entire process of evolution, the attenuation of $e_{\mathrm{t} z}$ will be much slower. From the physical sense, the

terms $P_{31}$ and $P_{35}$ can be regarded as a whole induced from the turn of $\omega_{y}^{\prime}$ by $\frac{\partial w}{\partial y}$, while the terms $P_{33}$ and $P_{36}$ can be taken as a whole which can be attributed to the stretch of $\omega_{z}$.

\subsection{Transport of $e_{\mathrm{t} y}$ and $e_{\mathrm{t} x}$}

In this section, the transport of $e_{\mathrm{t} y}$ and $e_{\mathrm{t} x}$ is mainly focused. The transport equation for the global $e_{\mathrm{t} y}$ can be written as

$$
\begin{aligned}
\frac{\partial\left(\omega_{y}^{\prime} \omega_{y}^{\prime}\right)}{\partial t}= & \underbrace{2\left(\omega_{y}^{\prime} \frac{\partial v^{\prime}}{\partial x} \frac{\partial \bar{w}}{\partial y}\right)}_{P_{21}}+\underbrace{2\left(-\omega_{y}^{\prime} \frac{\partial v^{\prime}}{\partial z} \frac{\partial \bar{u}}{\partial y}\right)}_{P_{22}}+\underbrace{2\left(\omega_{y}^{\prime} \omega_{x}^{\prime} \frac{\partial v^{\prime}}{\partial x}\right)}_{P_{23}} \\
& +\underbrace{2\left(\omega_{y}^{\prime} \omega_{y}^{\prime} \frac{\partial v^{\prime}}{\partial y}\right)}_{P_{24}}+\underbrace{2\left(\omega_{y}^{\prime} \omega_{z}^{\prime} \frac{\partial v^{\prime}}{\partial z}\right)}_{P_{25}}-\underbrace{2 \nu\left(\frac{\partial \omega_{y}^{\prime}}{\partial x_{j}} \frac{\partial \omega_{y}^{\prime}}{\partial x_{j}}\right)}_{D_{2}},
\end{aligned}
$$

where $P_{21}$ and $P_{22}$ are induced by the inclination of $\bar{\omega}_{x}$ and $\bar{\omega}_{z}$, while $P_{23}$ and $P_{25}$ are generated by the inclination of $\omega_{x}^{\prime}$ and $\omega_{z}^{\prime}$, respectively, and the term $P_{24}$ is generated by the stretching of $\omega_{y}^{\prime}$. Figure 8 shows the evolution of the production terms of $e_{\mathrm{t} y}$ in the initial stage after the SWO. Evidently, the suppression of $e_{\mathrm{t} y}$ is mainly dominated by the terms $P_{22}$ and $P_{24}$ which result from the inclination of $\bar{\omega}_{z}$ and the stretching of $\omega_{y}^{\prime}$, respectively. Different from the transport of $e_{\mathrm{t} z}$, the term $P_{21}$, which is directly related to the spanwise mean shear, only plays a secondary role here.

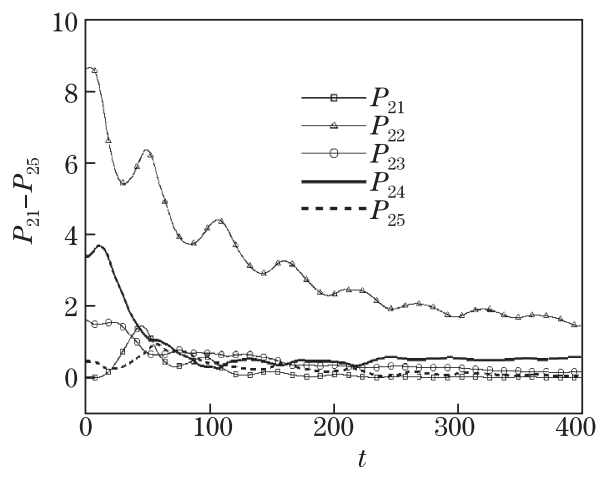

Fig. 8 Time traces of production terms of $e_{\mathrm{t} y}$ (SWO starts at $t=0$ ), where all terms are multiplied by scale factor of 100 
The transport equation for the global $e_{\mathrm{t} x}$ can be written as

$$
\begin{aligned}
\frac{\partial\left(\omega_{x}^{\prime} \omega_{x}^{\prime}\right)}{\partial t}= & \underbrace{2\left(\omega_{x}^{\prime} \omega_{y}^{\prime} \frac{\partial \bar{u}}{\partial y}\right)}_{P_{11}}+\underbrace{2\left(\omega_{x}^{\prime} \frac{\partial u^{\prime}}{\partial x} \frac{\partial \bar{w}}{\partial y}\right)}_{P_{12}}-\underbrace{2\left(\omega_{x}^{\prime} \frac{\partial u^{\prime}}{\partial z} \frac{\partial \bar{u}}{\partial y}\right)}_{P_{13}} \\
& +\underbrace{2\left(\omega_{x}^{\prime} \omega_{x}^{\prime} \frac{\partial u^{\prime}}{\partial x}\right)}_{P_{14}}+\underbrace{2\left(\omega_{x}^{\prime} \omega_{y}^{\prime} \frac{\partial u^{\prime}}{\partial y}\right)}_{P_{15}}+\underbrace{2\left(\omega_{x}^{\prime} \omega_{z}^{\prime} \frac{\partial u^{\prime}}{\partial z}\right)}_{P_{16}} \\
& -\underbrace{2\left(v^{\prime} \omega_{x}^{\prime} \frac{\partial^{2} \bar{w}}{\partial y^{2}}\right)}_{P_{17}}-\underbrace{2 \nu\left(\frac{\partial \omega_{x}^{\prime}}{\partial x_{j}} \frac{\partial \omega_{x}^{\prime}}{\partial x_{j}}\right)}_{D_{1}},
\end{aligned}
$$

where $P_{11}$ is generated from the inclination of $\omega_{y}^{\prime}$ by the streamwise mean shear, $P_{12}$ is the production term of $e_{\mathrm{t} x}$ from the contribution of the stretching of $\bar{\omega}_{x}, P_{13}$ is induced by the tilting of $\bar{\omega}_{z}$ by $\frac{\partial u^{\prime}}{\partial z}, P_{14}$ is the production term related to the stretching of $\omega_{x}^{\prime}, P_{15}$ and $P_{16}$ are induced by the turn of $\omega_{y}^{\prime}$ and $\omega_{z}^{\prime}$, respectively, and $P_{17}$ is the production term related to the second-order derivative of $\bar{w}$. The last term in Eq. (15), namely, $D_{1}$, is the global quantity of the viscous dissipation term. For clarity, the terms $P_{11}$ and $P_{13}$ can be regarded as a whole,

$$
\underbrace{2\left(\omega_{x}^{\prime} \omega_{y}^{\prime} \frac{\partial \bar{u}}{\partial y}\right)}_{P_{11}}-\underbrace{2\left(\omega_{x}^{\prime} \frac{\partial u^{\prime}}{\partial z} \frac{\partial \bar{u}}{\partial y}\right)}_{P_{13}}=\underbrace{\left(-\omega_{x}^{\prime} \frac{\partial w^{\prime}}{\partial x} \frac{\partial \bar{u}}{\partial y}\right)}_{P_{c 13}} .
$$

The terms $P_{15}$ and $P_{16}$ can be expanded as

$$
\begin{aligned}
& \underbrace{2\left(\omega_{x}^{\prime} \omega_{y}^{\prime} \frac{\partial u^{\prime}}{\partial y}\right)}_{P_{15}}=\underbrace{2\left(\omega_{x}^{\prime} \frac{\partial u^{\prime}}{\partial z} \frac{\partial u^{\prime}}{\partial y}\right)}_{15 a}-\underbrace{2\left(\omega_{x}^{\prime} \frac{\partial w^{\prime}}{\partial x} \frac{\partial u^{\prime}}{\partial y}\right)}_{15 b}, \\
& \underbrace{2\left(\omega_{x}^{\prime} \omega_{z}^{\prime} \frac{\partial u^{\prime}}{\partial z}\right)}_{P_{16}}=\underbrace{2\left(\omega_{x}^{\prime} \frac{\partial v^{\prime}}{\partial x} \frac{\partial u^{\prime}}{\partial z}\right)}_{16 a}-\underbrace{2\left(\omega_{x}^{\prime} \frac{\partial u^{\prime}}{\partial y} \frac{\partial u^{\prime}}{\partial z}\right)}_{16 b} .
\end{aligned}
$$

The order-of-magnitude analysis taken by Ricco et al. ${ }^{[19]}$ is adopted here. The length scale of the disturbance along $x$ can be taken as $\lambda_{x}=O(1000)$, namely, the characteristic length of the low-speed streaks, while the length scale of the distance along the $z$-axis can be taken as $\lambda_{z}=O(100)$, denoting that the characteristic spacing of the low-speed streaks. For the turbulent channel with the SWO, when $T=100$, the length scale along the $y$-axis can be assumed as $\lambda_{y}=O(10)$. Through the order-of-magnitude analysis, it is easy to get the results that the term $15 a$ is much larger than the term $15 b$ in Eq. (17), and the term $16 a$ is much smaller than $16 b$ in Eq. (18). Through a careful inspection of Eqs. (17) and (18), it is found that the main contributors of the two equations are opposite to each other. Hence, the terms $P_{15}$ and $P_{16}$ can be put together as a whole with only the secondary terms left.

The evolution of the production terms of $e_{\mathrm{t} x}$ is shown in Fig. 9. Compared with the evolutions of the $e_{\mathrm{t} y}$ and $e_{\mathrm{t} z}$, the evolution of $e_{\mathrm{t} x}$ seems a little slower, as shown in Fig. 4 . The term $P_{12}$ which directly relates with the SWO only plays a minor role in the evolution of $e_{\mathrm{t} x}$. Instead, the term $P_{c 13}$ combined with the term $P_{14}$ play as the dominate contributors. In the beginning $t<50$, a transient growth occurs on $e_{\mathrm{t} x}$, after that $e_{\mathrm{t} x}$ is suppressed. A similar evolution process can also be observed for the terms $P_{c 13}$ and $P_{14}$. Hence, both the transient growth of $e_{\mathrm{t} x}$ in the initial stage and the attenuation of $e_{\mathrm{t} x}$ after the beginning can be attributed to the term $P_{c 13}$ due to the horizontal shear of $\bar{\omega}_{z}$ and the term $P_{14}$ due to the stretching of $\omega_{x}^{\prime}$. 


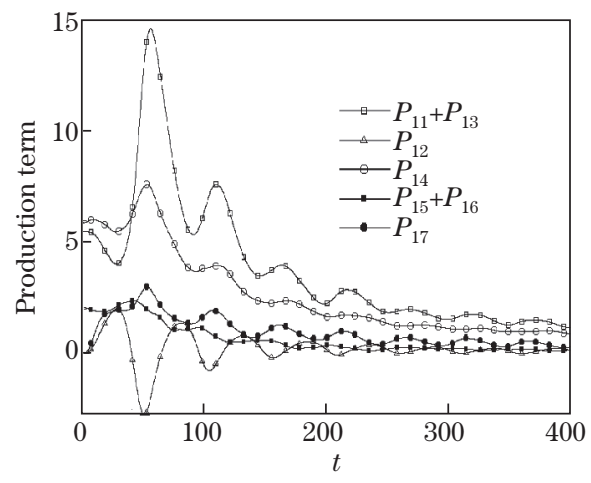

Fig. 9 Time traces of production terms of $e_{\mathrm{t} x}$ (SWO starts at $\left.t=0\right)$, where all terms are multiplied by scale factor of 100

\section{Summary and conclusions}

The transport of turbulent enstrophy after the SWO is studied in detail in a turbulent channel flow. The initial transient increase of the turbulent enstrophy as well as the suppression of the turbulent enstrophy after the beginning are focused, aiming to reveal the effect of the SWO in the aspect of vortical dynamics. For clarity, the turbulent enstrophy is divided into three parts: $\omega_{x}^{\prime} \omega_{x}^{\prime}\left(e_{\mathrm{t} x}\right), \omega_{y}^{\prime} \omega_{y}^{\prime}\left(e_{\mathrm{t} y}\right)$, and $\omega_{z}^{\prime} \omega_{z}^{\prime}\left(e_{\mathrm{t} z}\right)$.

The transport of $e_{\mathrm{t} z}$ is dominated by $P_{31}$ (contribution induced by the tilting of $\omega_{y}^{\prime}$ by the spanwise mean shear), $P_{33}$ (contribution from the stretching of $\bar{\omega}_{z}$ by the fluctuating quantity $\frac{\partial w^{\prime}}{\partial z}$ ), $P_{34}$ (contribution from the turn of $\omega_{x}^{\prime}$ ), and $P_{35}$ (contribution from the turn of $\omega_{y}^{\prime}$ ). Due to the SWO, a significant correlation is formed between $\omega_{y}^{\prime}$ and $\omega_{z}^{\prime}$, which induces the transit growth of the newly appearing term $P_{31}$ under the spanwise mean shear. The term $P_{31}$ then results in the transient growth of $e_{\mathrm{t} z}$ at the beginning, which in turn enhances the turbulent dissipation and drifts the turbulent flow towards a new lower-drag condition after a long time. Afterwards, both the turn of $\omega_{y}^{\prime}$ and the stretching of $\omega_{z}$ are suppressed, resulting in the attenuation of $e_{\mathrm{t} z}$.

The attenuation of $e_{\mathrm{t} y}$ in the initial stage can be ascribed to the terms related to the inclination of $\bar{\omega}_{z}$ and the stretching of $\omega_{y}^{\prime}$. Compared with the response of $e_{\mathrm{t} y}$ and $e_{\mathrm{t} z}$ to the SWO, the response of $e_{\mathrm{t} x}$ is much slower. The transient growth of $e_{\mathrm{t} x}$ in the initial stage as well as the attenuation of $e_{\mathrm{t} x}$ after the beginning of SWO can both be attributed to the change of the stretching of $\omega_{x}^{\prime}$ and the horizontal shear of $\bar{\omega}_{z}$ due to the SWO.

\section{References}

[1] Bechert, D. W., Bruse, M., Hage, W., van der Hoeven, J. G. T., and Hoppe, G. Experiments on drag-reducing surfaces and their optimization with an adjustable geometry. Journal of Fluid Mechanics, 338, 59-87 (1997)

[2] Garcia-Mayoral, R. and Jiménez, J. Hydrodynamic stability and breakdown of the viscous regime over riblets. Journal of Fluid Mechanics, 678, 317-347 (2011)

[3] Viswanath, P. R. Aircraft viscous drag reduction using riblets. Progress in Aerospace Sciences, 38(6), 571-600 (2002)

[4] Aljallis, E., Sarshar, M. A., Datla, R., Sikka, V., Jones, A., and Choi, C. H. Experimental study of skin friction drag reduction on super hydrophobic flat plates in high Reynolds number boundary layer flow. Physics of Fluids, 25(2), 025103 (2013) 
[5] Rothstein, J. P. Slip on superhydrophobic surfaces. Annual Review of Fluid Mechanics, 42(1), 89-109 (2010)

[6] Rastegari, A. and Akhavan, R. On the mechanism of turbulent drag reduction with superhydrophobic surfaces. Journal of Fluid Mechanics, 773, R4 (2015)

[7] Luo, Y., Wang, L., Lork, G., Song, K., Wang, L., and Robert, S. Advances of drag-reducing surface technologies in turbulence based on boundary layer control. Journal of Hydrodynamics, $\mathbf{2 7}(4)$, 473-487 (2015)

[8] Quadrio, M. and Ricco, P. Critical assessment of turbulent drag reduction through spanwise wall oscillations. Journal of Fluid Mechanics, 521(12), 251-271 (2004)

[9] Yakeno, A., Hasegawa, Y., and Kasagi, N. Modification of quasi-streamwise vortical structure in a drag-reduced turbulent channel flow with spanwise wall oscillation. Physics of Fluids, 26(8), 085109 (2014)

[10] Agostini, L., Touber, E., and Leschziner, M. A. Spanwise oscillatory wall motion in channel flow: drag-reduction mechanisms inferred from DNS-predicted phase-wise property variations at ReT $=1$ 000. Journal of Fluid Mechanics, 743, 606-635 (2014)

[11] Blesbois, O. and Chernyshenko, S. I. Pattern prediction by linear analysis of turbulent flow with drag reduction by wall oscillation. Journal of Fluid Mechanics, 724(2), 607-641 (2013)

[12] Jung, W. J., Mangiavacchi, N., and Akhavan, R. Suppression of turbulence in wall-bounded flows by high-frequency spanwise oscillations. Physics of Fluids, 4(8), 1605-1607 (1992)

[13] Laadhari, F., Skandaji, L., and Morel, R. Turbulence reduction in a boundary layer by a local spanwise oscillating surface. Physics of Fluids, 6(10), 3218-3220 (1994)

[14] Choi, K. S., Debisschop, J. R., and Clayton, B. R. Turbulent boundary-Layer control by means of spanwise-wall oscillation. AIAA Journal, 36(7), 1157-1163 (1998)

[15] Quadrio, M. and Sibilla, S. Numerical simulation of turbulent flow in a pipe oscillating around its axis. Journal of Fluid Mechanics, 424(14), 217-241 (2000)

[16] Choi, K. S. and Graham, M. Drag reduction of turbulent pipe flows by circular-wall oscillation. Physics of Fluids, 10(1), 7-9 (1998)

[17] Xu, C. X. and Huang, W. X. Transient response of Reynolds stress transport to spanwise wall oscillation in a turbulent channel flow. Physics of Fluids, 17(1), 018101 (2005)

[18] Quadrio, M. and Ricco, P. Initial response of a turbulent channel flow to spanwise oscillation of the walls. Journal of Turbulence, 4(7), 1-23 (2003)

[19] Ricco, P., Ottonelli, C., Hasegawa, Y., and Quadrio, M. Changes in turbulent dissipation in a channel flow with oscillating walls. Journal of Fluid Mechanics, 700, 77-104 (2012)

[20] Xu, C., Zhang, Z., Toonder, J. M. J. D., and Nieuwstadt, F. T. M. Origin of high kurtosis levels in the viscous sublayer, direct numerical simulation and experiment. Physics of Fluids, 8(7), 1938-1944 (1996)

[21] Ge, M., Xu, C., and Cui, G. Direct numerical simulation of flow in channel with time-dependent wall geometry. Applied Mathematics and Mechanics (English Edition), 31(1), 97-108 (2010) DOI 10.1007/s10483-010-0110-x

[22] Touber, E. and Leschziner, M. A. Near-wall streak modification by spanwise oscillatory wall motion and drag-reduction mechanisms. Journal of Fluid Mechanics, 693(2), 150-200 (2012)

[23] Kasagi, N., Hasegawa, Y., and Fukagata, K. Toward cost-effective control of wall turbulence for skin friction drag reduction. Advances in Turbulence, 52(1), 189-200 (2009)

[24] Ricco, P. and Wu, S. On the effects of lateral wall oscillations on a turbulent boundary layer. Experimental Thermal and Fluid Science, 29(1), 41-52 (2004)

[25] Choi, J. I., Xu, C. X., and Sung, H. J. Drag reduction by spanwise wall oscillation in wall-bounded turbulent flows. AIAA Journal, 40(5), 842-850 (2002) 